a s.

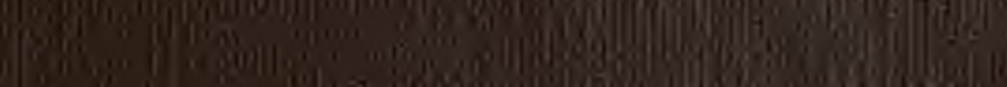

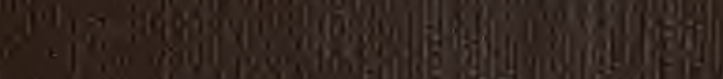

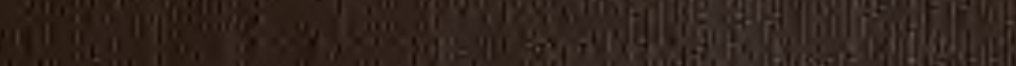

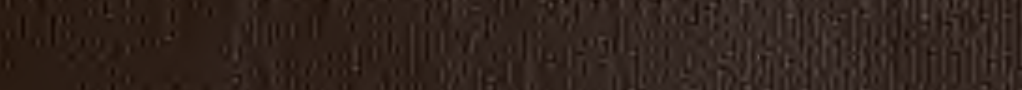

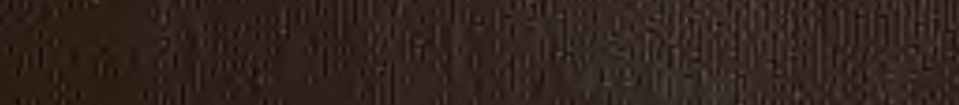

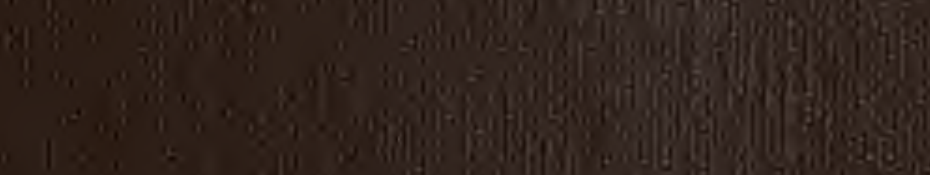

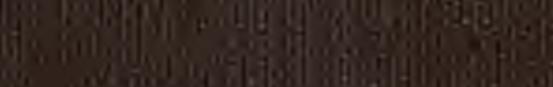
60.5. wive $=$, susing Dis

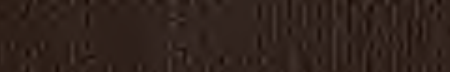

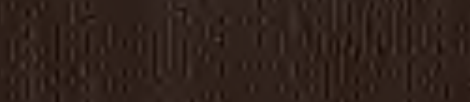
6.

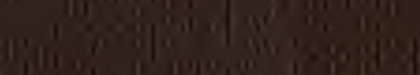

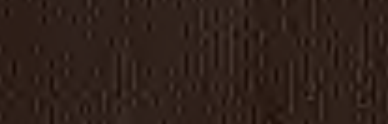
4. S18: 


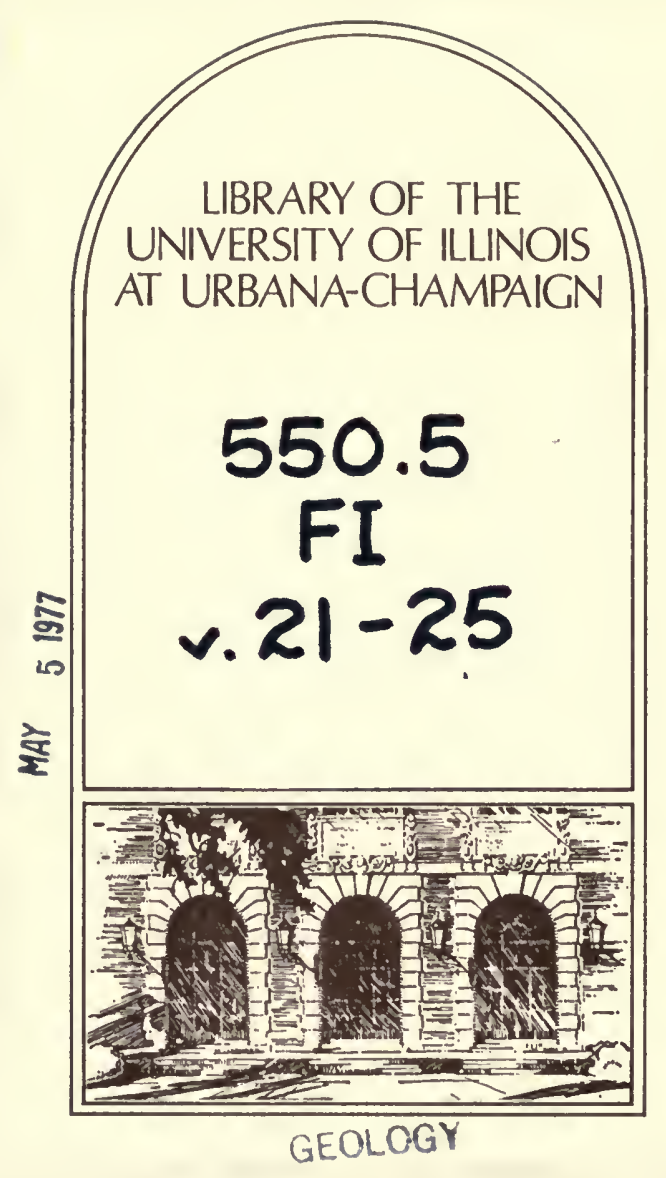

\author{
UNIVEASITY OF \\ ILLINOIS LIBRARY \\ AT URBANA-CHAMPAIGN \\ GEOLOGY
}






\section{FIELDIANA Geology}

\section{Catalogue of Type and Referred Specimens} of Crinozoa (Cystoidea) in

\section{Field Museum of Natural History}

\author{
Julia Golden \\ Assistant Editor, Micropaleontology Press \\ American Museum of Natural History \\ AND \\ MatThew H. Nitecki \\ Associate Curator, Fossil. Invertebrates \\ Field Museum of Natural History
}

\section{INTRODUCTION}

This catalogue lists the holotypes, referred specimens, and casts of types and of referred specimens of cystoids in the collection of Field Museum of Natural History. Only holotypes are recognized as types and all other socalled types and figured or described specimens are catalogued as referred specimens. The name of genus, species, and its authorship is entered in its original spelling and subsequent systematic changes are noted. Stratigraphic and geographic locations are given as those of the original authors. Specimens prefixed UC are formerly Walker Museum of Paleontology, University of Chicago, and specimens prefixed $\mathrm{P}$ or PE are Field Museum fossils.

This work was supported in part by the National Science Foundation Grant GB-7197.

\section{CATALOGUE OF TYPE AND REFERRED SPECIMENS}

Allocystites hammelli S. A. Miller, 1889

Holotype: UC 6006.

S. A. Miller, 1889, p. 222, text fig. 242.

Foerste, 1920, pp. 54-55, pl. 1, figs. 13A-B.

Kesling in Moore, 1967, p. 243, text figs. 143, 4a,4b. 
Silurian, Niagaran.

Jefferson County, Indiana; Gurley Collection.

Callocystites brevis Frest and Paul, 1971

Referred specimens: PE 19241-19246, 24 isolated plates.

Frest and Paul, 1971, pp. 425-431.

Silurian, Top $45 \mathrm{~cm}$. of Laurel Limestone (= Laurel Member of the Solomonie Formation).

Old abandoned quarry on the south side of Middle Branch, Clifty Creek, $0.63 \mathrm{~km}$. east of the junction of State Highway 3, and the county road to Clarksburg, in Sandusky, Decatur County. SE1/4, NE $1 / 4$, NW $1 / 4$, sec. 12, T.11N., R.9E., Milroy quadrangle, Indiana.

Caryocrinites bulbulus (S. A. Miller and Gurley, 1894)

See: Caryocrinus bulbulus S. A. Miller and Gurley, 1894.

Caryocrinites indianensis (S. A. Miller, 1891)

See: Caryocrinus indianensis S. A. Miller, 1891.

Caryocrinites kentuckiensis (S. A. Miller and Gurley, 1895)

See: Caryocrinus kentuckiensis S. A. Miller and Gurley, 1895.

Caryocrinus bulbulus S. A. Miller and Gurley, 1894

Holotype, not designated, to be selected from UC 6032.

Referred specimens: UC 6032, two specimens.

S. A. Miller and Gurley, 1894, pp. 11-12, pl. 2, figs. 15-18.

S. A. Miller, 1897, p. 740, text fig. 1324.

=Caryocrinites bulbulus (S. A. Miller and Gurley, 1894)

Bassler, 1915, p. 186.

=Stribalocystites bulbulus (S. A. Miller and Gurley, 1894)

Kesling in Moore, 1967, pp. 225-226.

Silurian, Niagaran.

Wayne County, Tennessee; Gurley Collection.

Caryocrinus indianensis S. A. Miller, 1891

Holotype: UC 6028. 
S. A. Miller, 1891, pp. 19-20, pl. 5, figs. 9, 10.

S. A. Miller, 1892a, pp. 629-630, pl. 5, figs. 9, 10.

S. A. Miller, 1892b, p. 676, text fig. 1218

=Caryocrinites indianensis (S. A. Miller, 1891)

Bassler, 1915, p. 187.

Silurian, Niagaran.

Jefferson County, Indiana; Gurley Collection.

Caryocrinus kentuckiensis S. A. Miller and Gurley, 1895

Holotype: UC 6127.

S. A. Miller and Gurley, 1895, pp. 59-60, pl. 5, figs. 22-24.

S. A. Miller, 1897, p. 740, text fig. 1320.

=Caryocrinites kentuckiensis (S. A. Miller and Gurley, 1895)

Bassler, 1915, p. 187.

Silurian, Niagara Group.

Louisville, Kentucky; Gurley Collection.

Coelocystis subglobosus (Hall, 1868)

Referred specimens: UC 22906, two specimens.

Foerste, 1920, pp. 40-43, pl. 1, figs. 1 A, B.

Shimer and Shrock, 1944, p. 128, pl. 48, figs. 9, 10.

Kesling in Moore, 1967, pp. 201-202, text figs. 101,1i, lj.

Silurian, Niagaran.

Chicago, Illinois; Hindshaw Collection.

Gomphocystites bownockeri Foerste, 1920

Referred specimens: UC 22943, two natural molds.

Foerste, 1920, pp. 57-59, pl. 1, figs. 6A, B.

Kesling in Moore, 1967, p. 240, text fig. 140,1g.

Silurian, Racine Dolomite.

Chicago, Illinois; Hindshaw Collection.

Referred specimens: UC 22944, three specimens.

Foerste, 1920, pp. 57-59.

Silurian, Niagaran.

Chicago, Illinois; Hindshaw Collection.

Referred specimen: UC 22945.

Foerste, 1920, pp. 57-59, pl. 1, figs. 9A, B. 
Silurian, Niagaran.

Bridgeport Quarry, Chicago, Illinois; Hindshaw Collection.

Gomphocystites indianensis S. A. Miller, 1889

Holotype: UC 6019.

S. A. Miller, 1889, p. 249, text fig. 319.

Foerste, 1920, pp. 55-56, pl. 1, figs. 5A, B.

Kesling in Moore, 1967, p. 240, text figs. 140,1i,1j.

Silurian, Niagaran.

Jefferson County, Indiana; Gurley Collection.

Hallicystis imago (Hall, 1864)

Referred specimen: UC 21734B.

Foerste, 1920, p. 39, pl. 1, fig. 8.

Kesling in Moore, 1967, p. 209, text fig. 105,2c.

Silurian, Niagaran.

Racine, Wisconsin; Van Horne Collection.

Referred specimens: UC 22908, two specimens.

Foerste, 1920, p. 39, pl. 1, fig. 7.

Kesling in Moore, 1967, p. 209, text fig. 105,2b.

Silurian, Niagaran.

Chicago; collected by W. R. Head.

Holocystites abnormis (Hall, 1864)

See: Holocystites canneus S. A. Miller, 1889.

Holocystites adipatus S. A. Miller, 1891

Holotype: UC 6020.

S. A. Miller, 1891, p. 13, pl. 2, figs. 1, 2.

S. A. Miller, 1892a, p. 623, pl. 2, figs. 1, 2.

$=$ Triamara tumida $(\mathrm{S}$. A. Miller, 1879)

Paul, 1971, pp. 58-63, text figs. 14A, 18B.

Silurian, Niagara Group, lower part.

Jefferson County, Indiana; Gurley Collection.

Holocystites affinis S. A. Miller and Faber, 1892

Holotype: UC 8761. 
S. A. Miller and Faber, 1892, p. 87, pl. 1, figs. 16-18.

= Pustulocystis ornatissimus $(\mathrm{S}$. A. Miller, 1891)

Paul, 1971, pp. 133-138, text fig. 56G.

Silurian, Niagara Group.

Near Madison, Indiana; Faber Collection.

Holocystites alternatus (Hall, 1861)

See: Holocystites baculus S. A. Miller, 1879

Holocystites colletti S. A. Miller, 1891

Holocystites perlongus S. A. Miller, 1878

Holocystites amplus S. A. Miller, 1892

Holotype: UC 6021.

S. A. Miller, 1892c, pp. 8-9, pl. 2, fig. 1.

S. A. Miller, 1894, pp. 262-263, pl. 2, fig. 1.

=Triamara ventricosa $(\mathrm{S}$. A. Miller, 1879)

Paul, 1971, pp. 54-58, text figs. 14D, 15A, 15B.

Silurian, Niagara Group.

Near Madison, Indiana; Gurley Collection.

Holocystites asper S. A. Miller and Gurley, 1895

Holotype: UC 6001.

S. A. Miller and Gurley, 1895b, pp. 84-85, pl. 5, figs. 1, 2.

$=$ Pustulocystis ornatissimus (S. A. Miller, 1891)

Paul, 1971, pp. 133-138, text figs. 57A, 57B.

Silurian, Niagara Group, lower part.

Near Madison, Indiana; Gurley Collection.

Holocystites baculus S. A. Miller, 1879

Holotype: UC 8762.

S. A. Miller, 1879, pp. 105-106, pl. 10, figs. 5, 5A.

$=$ Holocystites alternatus (Hall, 1861)

Paul, 1971, pp. 83-89, text fig. 31.

Silurian, Niagara Group.

Osgood, Ripley County, Indiana; Faber Collection.

Holocystites benedicti S. A. Miller, 1891

Holotype: UC 6015. 
S. A. Miller, 1891, p. 17, pl. 5, fig. 3.

S. A. Miller, 1892a, p. 627, pl. 5, fig. 3.

="Holocystites" benedicti S. A. Miller, 1891

Paul, 1971, pp. 154-155, text fig. 70.

Silurian, Niagara Group.

Jefferson County, Indiana; Gurley Collection.

Holocystites canneus S. A. Miller, 1889

Holotype: UC 6002.

S. A. Miller, 1889, pp. 253-254, text figs. 332, 333.

= Holocystites abnormis (Hall, 1864)

Paul, 1971, pp. 89-92, text figs. 33A, 33B.

Silurian, Niagara Group.

Jefferson County, Indiana; Gurley Collection.

Holocystites colletti S. A. Miller, 1891

Holotype: UC 8768.

S. A. Miller, 1891, p. 16, pl. 4, fig. 3.

S. A. Miller, 1892a, p. 626, pl. 4, fig. 3.

=Holocystites alternatus (Hall, 1861)

Paul, 1971, pp. 83-89, text fig. 29D.

Silurian, Niagara Group.

Jefferson County, Indiana; Gurley Collection.

Holocystites commodus S. A. Miller, 1891

Lectotype: designated by Paul, 1971, p. 127, UC 6018A.

S. A. Miller, 1891, p. 14, pl. 3, figs. 1, 2.

S. A. Miller, 1892a, p. 624, pl. 3, figs. 1, 2.

$=$ Trematocystis rotundus $(\mathrm{S}$. A. Miller, 1879)

Paul, 1971, pp. 124-129, text figs. 52B, 53A, 53B.

Referred specimen: UC 6018B.

S. A. Miller, 1891, p. 14, pl. 3, figs. 5, 6.

S. A. Miller, 1892a, p. 624, pl. 3, figs. 5, 6.

$=$ Trematocystis rotundus $(\mathrm{S}$. A. Miller, 1879)

Paul, 1971, pp. 124-129, text fig. 53C.

Silurian, Niagara Group.

Jefferson County, Indiana; Gurley Collection. 
Holocystites cylindricus (Hall, 1861)

Referred specimens: P 8462A-B, two specimens.

Paul, 1971, pp. 71, 79-83, text figs. 27E (P 8462B), 28A (P 8462A).

Silurian, Joliet Dolomite.

Lemont, Illinois; collected by Slocom.

Referred specimen: P 10533.

Paul, 1971, pp. 79-83, text fig. 28B.

Silurian, Joliet Dolomite.

Romeo, Illinois.

Holocystites faberi S. A. Miller, 1889

Holotype: UC 8764.

S. A. Miller, 1889 , p. 254 , text figs. $334,335$.

=Holocystites scutellatus Hall, 1864

Paul, 1971, pp. 94-101, text fig. 37A.

Silurian, Niagara Group.

Jeffers on County, Indiana; Faber Collection.

Holocystites globosus S. A. Miller, 1878

Lectotype: designated by Paul, 1971, p. 120, UC 8765A.

S. A. Miller, 1878, pp. 133-134, pl. 5, fig. 5.

$=$ Trematocystis globosus $($ S. A. Miller, 1878)

Paul, 1971, pp. 120-124, text figs. 48A, 48B.

Referred specimens: UC 8765B-C, two specimens.

S. A. Miller, 1878, pp. 133-134.

$=$ Trematocystis globosus $(\mathrm{S}$. A. Miller, 1878)

Paul, 1971, pp. 120-124.

Silurian, Niagara Group, lower part.

Jefferson County, Indiana; Faber Collection.

Holocystites gorbyi S. A. Miller, 1891

Holotype: UC 6014.

S. A. Miller, 1891, p. 14, pl. 2, figs. 3, 4.

S. A. Miller, 1892a, p. 624, pl. 2, figs. 3, 4.

$=$ Trematocystis rotundus $(\mathrm{S}$. A. Miller, 1879)

Paul, 1971, pp. 124-129, text fig. 53E.

Silurian, Niagara Group. 
Jefferson County, Indiana; Gurley Collection.

Holocystites gyrinus S. A. Miller and Gurley, 1894

Holotype: UC 5997.

S. A. Miller and Gurley, 1894, pp. 5-7, pl. 1, figs. 1-3.

S. A. Miller, 1897, p. 747 , text fig. 1361 .

=Triamara ventricosa $(\mathrm{S}$. A. Miller, 1879)

Paul, 1971, pp. 54-58, text figs. 14B, 17A, 17B.

Silurian, Niagara Group, lower part.

Madison, Jefferson County, Indiana; Gurley Collection.

Holocystites hammelli S. A. Miller, 1889

Holotype: UC 5999.

S. A. Miller, 1889, p. 254, text figs. 336, 337.

$=$ Trematocystis globosus $(\mathrm{S}$. A. Miller, 1878)

Paul, 1971, pp. 120-124, text figs. 48C, 48D.

Silurian, Niagara Group.

Jefferson County, Indiana; Gurley Collection.

Holocystites indianensis S. A. Miller, 1891

Holotype: UC 6016.

S. A. Miller, 1891 , p. 15 , pl. 3, fig. 7.

S. A. Miller, 1892a, p. 625, pl. 3, fig. 7.

$=$ Trematocystis rotundus $(\mathrm{S}$. A. Miller, 1879)

Paul, 1971, pp. 124-129, text fig. 53D.

Silurian, Niagara Group, lower part.

Jefferson County, Indiana; Gurley Collection.

Holocystites madisonensis S. A. Miller, 1891

Holotype: UC 6017.

S. A. Miller, 1891, p. 15, pl. 3, figs. 3, 4.

S. A. Miller, 1892a, p. 625, pl. 3, figs. 3, 4.

=“Holocystites" madisonensis S. A. Miller, 1891

Paul, 1971, p. 154, text fig. 69.

Silurian, Niagara Group, lower part.

Jefferson County, Indiana; Gurley Collection.

Holocystites ornatissimus S. A. Miller, 1891

Holotype: UC 8760. 
S. A. Miller, 1891, p. 17, pl. 5, figs. 1, 2.

S. A. Miller, 1892a, p. 627, pl. 5, figs. 1, 2.

Bather, 1919, p. 257, pl. 6, fig. 25.

=Pustulocystis ornatissimus (S. A. Miller, 1891)

Paul, 1971, pp. 133-138, text figs. 56A, 56B.

Silurian, Niagara Group.

Jefferson County, Indiana; Faber Collection.

Holocystites papulosus S. A. Miller, 1891

Holotype: UC 6011.

S. A. Miller, 1891, p. 18, pl. 5, figs. 7, 8.

S. A. Miller, 1892a, p. 628, pl. 5, figs. 7, 8.

=Pustulocystis ornatissimus (S. A. Miller, 1891)

Paul, 1971, pp. 133-138, text figs. 56C, 56D, 57C.

Silurian, Niagara Group.

Jefferson County, Indiana; Gurley Collection.

Holocystites parvulus S. A. Miller, 1891

Holotype: UC 6009.

S. A. Miller, 1891, p. 18, pl. 5, fig. 6.

S. A. Miller, 1892a, p. 628, pl. 5, fig. 6.

=Holocystites scutellatus Hall, 1864

Paul, 1971, pp. 94-101, text fig. 36C.

Silurian, Niagara Group.

Jefferson County, Indiana; Gurley Collection.

Holocystites parvus S. A. Miller, 1891

Holotype: UC 6010.

S. A. Miller, 1891, p. 16, pl. 4, figs. 4, 5.

S. A. Miller, 1892a, p. 626, pl. 4, figs. 4, 5.

$=$ Trematocystis rotundus $(\mathrm{S}$. A. Miller, 1879)

Paul, 1971, pp. 124-129, text fig. 52E.

Silurian, Niagara Group.

Jefferson County, Indiana; Gurley Collection.

Holocystites perlongus S. A. Miller, 1878

Holotype: UC 6007.

S. A. Miller, 1878, pp. 132-133, pl. 5, figs. 4, 4A. 
=Holocystites alternatus (Hall, 1861)

Paul, 1971, pp. 83-89, text fig. 30C.

Silurian, Niagara Group, lower part.

Osgood, Ripley County, Indiana; Gurley Collection.

Holocystites pustulosus S. A. Miller, 1878

Holotype: UC 2612.

S. A. Miller, 1878, pp. 134-135, pl. 6, figs. 1, la.

= Holocystites scutellatus Hall, 1864

Paul, 1971, pp. 94-101, text fig. 36F.

Silurian, Niagara Group.

A few miles from Waldron, Indiana; Washburn Collection.

Holocystites rotundus S. A. Miller, 1879

Holotype: original designation UC 6004.

S. A. Miller, 1879, p. 107, pl. 9, figs. 3-3B.

$=$ Trematocystis rotundus $(\mathrm{S}$. A. Miller, 1879)

Paul, 1971, pp. 124-129, text figs. 52A, 52C, 52D.

Silurian, Niagara Group.

Osgood, Ripley County, Indiana; Gurley Collection.

Holocystites scitulus S. A. Miller, 1891

Holotype: UC 6012.

S. A. Miller, 1891, p. 14, pl. 2, figs. 5, 6 .

S. A. Miller, 1892a, p. 624, pl. 2, figs. 5, 6.

S. A. Miller, 1897, p. 747, text fig. 1359.

$=$ Trematocystis globosus (S. A. Miller, 1878)

Paul, 1971, pp. 120-124, text figs. 48F, 48G.

Silurian, Niagara Group, lower part.

Jefferson County, Indiana; Gurley Collection.

Holocystites scutellatus Hall, 1864

Referred specimen: UC 10960.

Paul, 1971, pp. 94-101, text fig. 37F.

Silurian, Niagaran.

Jefferson County, Indiana.

See also: Holocystites faberi S. A. Miller, 1889.

Holocystites parvulus S. A. Miller, 1891. 
Holocystites pustulosus S. A. Miller, 1878.

Holocystites splendens S. A. Miller and Gurley, 1894.

Holocystites spangleri S. A. Miller, 1891

Holotype: UC 6008.

S. A. Miller, 1891, pp. 16-17, pl. 4, fig. 6.

S. A. Miller, 1892a, pp. 626-627, pl. 4, fig. 6.

Paul, 1971, pp. 93-94, text figs. 35A, 35B.

Silurian, Niagara Group.

Jefferson County, Indiana; Gurley Collection.

Remarks: Paul, 1971, p. 93, gives type locality and horizon as lower limestone along Big Creek, Indiana.

Referred specimen: UC 10963

Paul, 1971, p. 94.

Silurian, Niagara Group.

Madison, Indiana; Gurley Collection.

Holocystites sphaeroidalis S. A. Miller and Gurley, 1895

Holotype: UC 6000.

S. A. Miller and Gurley, 1895b, pp. 85-86, pl. 5, figs. 3, 4.

=Pentacystis sphaeroidalis (S. A. Miller and Gurley, 1895)

Paul, 1971, pp. 108-111, text figs. 41C, 43A-43C, 44A.

Silurian, Niagara Group.

Near Madison, Indiana; Gurley Collection.

Holocystites splendens S. A. Miller and Gurley, 1894

Holotype: UC 5998.

S. A. Miller and Gurley, 1894, pp. 7-8, pl. 1, figs. 7-9.

S. A. Miller, 1897, p. 747, text fig. 1360.

=Holocystites scutellatus Hall, 1864

Paul, 1971, pp. 94-101, text figs. 36A, 36B.

Silurian, Niagara Group.

Near Madison, Indiana; Gurley Collection.

Holocystites subglobosus S. A. Miller, 1889

Lect otype: designated by Paul, 1971, pp. 120-122, UC 8766A.

S. A. Miller, 1889, p. 255, text fig. 338.

$=$ Trematocystis globosus $(\mathrm{S}$. A. Miller, 1878) 
Paul, 1971, pp. 120-124, text figs. 48E, 48H, 49A.

Referred specimen: UC $8766 \mathrm{~B}$.

S. A. Miller, 1889, p. 255.

= Trematocystis globosus (S. A. Miller, 1878)

Paul, 1971, pp. 120-124

Silurian, Niagara Group.

Jeffers on County, Indiana; Faber Collection.

Holocystites subovatus S. A. Miller, 1891

Holotype: UC 6013.

S. A. Miller, 1891 , pp. $17-18$, pl. 5, figs. 4, 5.

S. A. Miller, 1892a, pp. 627-628, pl. 5, figs. 4, 5.

S. A. Miller, 1892b, p. 680, text fig. 1237 .

=Pustulocystis ornatissimus (S. A. Miller, 1891)

Paul, 1971, pp. 133-138, text figs. $56 \mathrm{E}, 56 \mathrm{~F}$.

Silurian, Niagara Group.

Jefferson County, Indiana; Gurley Collection.

Remarks: Paul, 1971, text figs. 56E and 56F, incorrectly lists specimen as "H." subonatus FMNH 6015.

Holocystites subrotundus S. A. Miller, 1879

Holotype: UC 6003.

S. A. Miller, 1879, pp. 107-108, pl. 9, figs. 2, 2a.

="Holocystites" subrotundus S. A. Miller, 1879

Paul, 1971, pp. 153-154, text fig. 68.

Silurian, Niagara Group, lower part.

Osgood, Ripley County, Indiana; Gurley Collection.

Holocystites tumidus S. A. Miller, 1879

Holotype: UC 8763.

S. A. Miller, 1879, pp. 104-105, pl. 9, figs. 1, 1a.

= Triamara tumida (S. A. Miller, 1879)

Paul, 1971, pp. 58-63, text figs. 14C, 18C.

Silurian, Niagara Group.

Osgood, Ripley County, Indiana; Faber Collection.

Holocystites ventricosus S. A. Miller, 1879

Holotype: UC 8767. 
S. A. Miller, 1879, pp. 108-109, pl. 10, fig. 4.

$=$ Triamara ventricosa $(\mathrm{S}$. A. Miller, 1879)

Paul, 1971, pp. 54-58, text fig. 15D.

Silurian, Niagara Group, lower part.

Osgood, Ripley County, Indiana; Faber Collection.

Holocystites wetherbyi S. A. Miller, 1878

Holotype: UC 6005.

S. A. Miller, 1878, pp. 131-132, pl. 5, figs. 2-2b.

$=$ Trematocystis wetherbyi $(\mathrm{S}$. A. Miller, 1878)

Paul, 1971, pp. 129-131, text figs. 55A, 55B.

Silurian, Niagara Group, lower part.

Osgood, Ripley County, Indiana; Gurley Collection.

Holocystites wykoffi S. A. Miller, 1891

Holotype: UC 6022.

S. A. Miller, 1891, p. 15, pl. 4, figs. 1, 2.

S. A. Miller, 1892a, p. 625, pl. 4, figs, $1,2$.

=Pentacystis wykoffi (S. A. Miller, 1891)

Paul, 1971, pp. 111-115, text figs. 41 B, 45A-45C, 46.

Silurian, Niagara Group.

Jefferson County, Indiana; Gurley Collection.

\section{Holocystites s.s.}

Referred specimen: UC 10965.

Paul, 1971, p. 74, text figs. 25A, 25B.

Silurian, Niagaran.

Jefferson County, Indiana; Gurley Collection.

Lepadocystis moorei (Meek, 1871)

Referred specimens: UC $9961 \mathrm{~A}$ and C, two specimens.

Foerste, 1914, pp. 459-468, text fig. 6, pl. 5, figs. 1A, 1C.

Ordovician, Cincinnatian, Richmond, Whitewater.

Richmond, Indiana; Faber Collection.

Pentacystis sphaeroidalis (S. A. Miller and Gurley, 1895)

Referred specimen: UC 10957A.

Paul, 1971, p. 108. 
Silurian, Niagaran.

Jefferson County, Indiana.

See also: Holocystites sphaeroidalis S. A. Miller and Gurley, 1895

Pentacystis wykoffi (S. A. Miller, 1891)

See: Holocystites wykoffi S. A Miller, 1891.

\section{Pleurocystites beckeri Foerste, 1924}

Holotype: original designation UC 27236.

Foerste in Slocom and Foerste, 1924, pp. 359-362, pl. 31, fig. 12, pl. 33, fig. 1, pl. 34, figs. 1A-D.

Ordovician, Lower Maquoketa Shale.

Clermont, lowa; gift of A. G. Becker.

Referred specimen: P 16884.

Foerste in Slocom and Foerste, 1924, pp. 359-362, pl. 33, fig. 6.

Ordovician, Lower Maquoketa Shale.

Clermont, lowa; collected by Slocom, 1912.

\section{Pleurocystites clermontensis Foerste, 1924}

Holotype: original designation P 16858.

Foerste in Slocom and Foerste, 1924, pp. 363-365, pl. 33, fig. 7.

Ordovician, Lower Maquoketa Shale.

Clermont, lowa; collected by Slocom, 1912.

Pleurocystites mercerensis S. A. Miller and Gurley, 1895

Holotype: UC 6047.

S. A. Miller and Gurley, 1895a, pp. 60-6I, pl. 5, figs. 25, 26.

S. A. Miller, 1897, p. 751, text fig. 1379.

Ordovician, Trenton.

Mercer County, Kentucky; Gurley Collection.

Pleurocystites slocomi Foerste, 1924

Holotype: original designation P 18529 [not P 16858].

Foerste in Slocom and Foerste, 1924, pp. 362-363, pl. 33, fig. 5, pl. 34, figs. 5A-D.

Ordovician, Lower Maquoketa Shale.

Clermont, Iowa; collected by Slocom, 1912. 


\section{Pleurocystites sp.}

Referred specimen: UC 52008.

Foerste in Slocom and Foerste, 1924, pp. 365-366, pl. 33, fig. 4. Ordovician, Middle Maquoketa.

Dover Township, Fayette County, lowa; gift of A. G. Becker.

Pustulocystis ornatissimus (S. A. Miller, 1891)

See: Holocystites affinis S. A. Miller and Faber, 1892.

Holocystites asper S. A. Miller and Gurley, 1895.

Holocystites ornatissimus S. A. Miller, 1891.

Holocystites papulosus S. A. Miller, 1891.

Holocystites subovatus S. A. Miller, 1891.

Stribalocystites bulbulus (S. A. Miller and Gurley, 1894)

See: Caryocrinus bulbulus S. A. Miller and Gurley, 1894.

Stribalocystites sphaeroidalis S. A. Miller and Gurley, 1895

Holotype: UC 6128.

S. A. Miller and Gurley, 1895a, pp. 58-59, pl. 5, figs. 19-21.

S. A. Miller, 1897, p. 753, text fig. 1397.

Silurian, Niagaran.

St. Paul, Indiana; Gurley Collection.

Trematocystis globosus (S. A. Miller, 1878)

Sec: Holocystites globosus S. A. Miller, 1878.

Holocystites hammelli S. A. Miller, 1889.

Holocystites scitulus S. A. Miller, 1891.

Holocystites subglobosus S. A. Miller, 1889.

Trematocystis rotundus (S. A. Miller, 1879)

Sce: Holocystites commodus S. A. Miller, 1891.

Holocystites gorbyi S. A. Miller, 1891.

Holocystites indianensis S. A. Miller, 1891.

Holocystites parvus S. A. Miller, 1891.

Holocystites rotundus S. A. Miller, 1879.

Trematocystis wetherbyi (S. A. Miller, 1878)

See: Holocystites wetherbyi S. A. Miller, 1878. 
Triamara cutleri Tillman, 1967

Holotype: original designation PE 10954.

Tillman, 1967, pp. 222-226, text figs. 1A-1E, pl. 25, figs. 1-7.

$=$ Triamara ventricosa $(\mathrm{S}$. A. Miller, 1879)

Paul, 1971, pp. 54-58, text figs. 1I, 15C, 16D-16F.

Silurian (Llandovery), Osgood Formation

Napoleon Lime Industry Company quarry, Napoleon, Indiana.

Triamara multiporata Paul, 1971

Holotype: original designation UC 15217.

Paul, 1971, pp. 63-65, text figs. 14E, 20A-20C.

Silurian, Osgood Beds.

Eight miles north of Madison, Jefferson County, Indiana.

Triamara tumida (S. A. Miller, 1879)

Referred specimen: P 8424A. [Not P 8428a].

Paul, 1971, pp. 58-63, text fig. 19B.

Silurian, Joliet Dolomite.

Romeo, Illinois; A. W. Slocom Collection.

See also: Holocystites adipatus S. A. Miller, 1891.

Holocystites tumidus S. A. Miller, 1879.

Triamara ventricosa (S. A. Miller, 1879)

Referred specimen: P 8423A.

Paul, 1971, pp. 54-58, text figs. 16A, 16B.

Silurian, Joliet Dolomite.

Romeo, Illinois; A. W. Slocom Collection.

See also: Holocystites amplus S. A. Miller, 1892.

Holocystites gyrinus S. A. Miller and Gurley, 1894.

Holocystites ventricosus S. A. Miller, 1879.

Triamara cutleri Tillman, 1967.

\section{CATALOGUE OF CASTS OF TYPE AND REFERRED SPECIMENS}

Caryocrinites ornatus Say, 1825

See: Caryocrinus ornatus (Say, 1825) 
Caryocrinus ornatus (Say, 1825)

Referred specimen: UC 54087.

Hall, 1843, p. 112, text figs. 41,1, 41,2. Table figs. 17,1, 17,1a.

Hall, 1852a, pp. 216-227, pl. 49, figs. 1b, lc.

Hall, 1852b, pp. 40-51, pl. 49, figs. 1b, 1c.

Lesley, 1889, p. 119, text figs.

Grabau, 1901, pp. 150-151, text fig. 46.

Grabau and Shimer, 1910, p. 465, text figs. 1772, 1773.

=Caryocrinites ornatus Say, 1825

Shimer and Shrock, 1944, p. 127, pl. 47, figs. 22, 23.

Kesling in Moore, 1967, pp. 223-225, text figs. 122, la, 122, 1 b.

Silurian, Niagaran.

Lockport, New York; Hall Collection.

Gomphocystites glans Hall, 1864

Referred specimen: UC 23327.

Hall, 1864, preprint of Hall, 1868 [Not seen].

Hall, 1868, p. 310, pl. 12(3), fig. 14.

Hall, 1870, pp. 352-353, pl. 12, fig. 14.

Chamberlin, 1883, p. 191, text fig. 51a.

Grabau and Shimer, 1910, p. 462, text fig. 1768.

Silurian, Niagaran.

Racine, Wisconsin; Hall Collection.

Remarks: UC 23327 is a cast of one of the specimens originally figured by Hall, 1864. It is not known whether a holotype has been designated.

Holocystites cylindricus (Hall, 1861)

Referred specimen: UC 54071.

Hall, 1864, preprint of Hall, 1868 [Not seen].

Hall, 1868, pp. 311-312, pl. 12(3), fig. 6, pl. 12a(1), fig. 8 .

Hall, 1870 , p. 354 , pl. 12, fig. 6, pl. 12a, fig. 8.

Chamberlin, 1883, p. 191, text fig. $51 \mathrm{f}$.

Paul, 1971, pp. 79-83, text fig. 27A.

Silurian.

Racine and Waukesha, Wisconsin.

Lepadocrinus (Apiocystites) gebhardi (Conrad, 1840)

See: Lepadocrinus gebhardi (Conrad, 1840). 
Lepadocrinus gebhardi (Conrad, 1840)

Referred specimen: UC 15844.

Hall, 1859, pp. 127-129.

Hall, 1861, pl. 7, figs. 1-4.

=Lepadocrinus (Apiocystites) gebhardi (Conrad, 1840)

Ward, 1866, p. 178, no. 936, text fig.

$=$ Lepocrinites gebhardii $($ Conrad, 1840)

Schuchert, 1913, pp. 229-231, pl. 32, figs. 4-6.

Shimer and Shrock, 1944, p. 128, pl. 48, fig. 5.

Kesling in Moore, 1967, pp. 211-212, text figs. 104,1a, 104,1 b.

Devonian [now Silurian], Manlius Beds.

Schoharie, New York; Hall Collection.

\section{Lepocrinites gebhardii Conrad, 1840}

See: Lepadocrinus gebhardi (Conrad, 1840).

\section{REFERENCES}

BASSLER, R. S.

1915. Bibliographic index of American Ordovician and Silurian fossils. 2 vols. Bull. U.S. Nat. Mus., 92, 1521 pp.

BATHER, F. A.

1919. 111.--Notes on Yunnan Cystidea. 111. Sinocystis compared with similar genera. Geol. Mag., 56, pp. 255-262, pl. 6.

Cilamberlin, T. C.

1883. Upper Silurian Age, Chapter 9, pp. 178-200, text figs. 44-58. In Geology of Wisconsin Survey of 1873-1879, 1, 725 pp., 157 text figs., 11 pls.

Foerste, Aug. F.

1914. Notes on Agelacrinidae and Lepadocystinae, with descriptions of Thresherodiscus and Brockocystis. Bull. Denison Univ. Sci. Lab., 17, pp. 399-487, pls. 1-6, 8 text figs.

1920. Racine and Cedarville cystids and blastoids with notes on other echinoderms. Ohio Jour. Sci., 21, no. 2, pp. 33-78, pls. 1-4, 4 text figs.

Frist, T. and C. R. C. Paul

1971. Callocistites brevis, a new Silurian rhombiferan cystoid from Indiana. Geol. Mag., 108, no. 5, pp. 425-432, I pl., 4 text-figs., I table.

Graba!, Amadeus W.

1901. Guide to the Geology and Paleontology of Niagara Falls and vicinity. Bull. New York State Mus., 9, no. 45, 284 pp., 190 text figs.

Grabal, Amadeus W. and Hervey Woodburn Shimer

1910. North American Index Fossils. Invertebrates, vol. 2. New York. 909 pp., text figs. 1211-1937. 
HALL, J AMES

1843. Natural History of New York. Part 4. Geology of New York. Comprising the survey of the fourth geological district, 683 pp., 192 text figs., 74 tables of organic remains, $19 \mathrm{pls}$.

1852a. Natural History of New York. Part 6. Palaeontology of New York, 2, containing descriptions of the organic remains of the Lower Middle Division of the New York System, 363 pp., 109 pls., several text figs.

1852b. Crinoideae of the Clinton and Niagara Groups. Extract from Palaeontology of New York, 2, 72 pp., pls. $41 \mathrm{~A}-51$.

1859. Natural History of New York. Part 6. Palaeontology of New York, 3, containing descriptions and figures of the organic remains of the Lower Helderberg Group and the Oriskany Sandstone, part 1, text, $532 \mathrm{pp}$.

1861. Natural History of New York. Part 6. Palaeontology of New York, 3, containing descriptions and figures of the organic remains of the Lower Helderberg Group and the Oriskany Sandstone, part 2, plates, 120 pls.

1864. A preprint of Hall, 1868, published in advance for the 18th Ann. Rept., New York State Cab. Nat. Hist. [Not seen].

1868. Account of some new or little-known species of fossils from rocks of the age of the Niagara Group. 20th Ann. Rept., New York State Cab. Nat. Hist., pp. 305-401, pls. 9-22.

1870. Descriptions of new or little-known species of fossils from rocks of the age of the Niagara Group. 20th Ann. Rept., New York State Cab. Nat. Hist., revised edition, pp. $347-434$, pls. $9-25$.

LESLEY, J. P.

1889. A dictionary of the fossils of Pennsylvania and neighboring states named in the reports and catalogues of the survey. Geol. Surv. Pennsylvania Rept., P4, vol. 1 A-M, 437 pp., numerous text figs.

MiLleR, S. A.

1878. Description of eight new species of Holocystites from the Niagara Group. Jour. Cincinnati Soc. Nat. Hist., 1, no. 3, pp. 129-136, pls. 5 and part of 6.

1879. Description of twelve new fossil species, and remarks upon others. Jour. Cincinnati Soc. Nat. Hist., 2, no. 2, pp. 104-118, pls. 9-10.

1889. North American geology and palaeontology for the use of amateurs, students, and scientists. Cincinnati, Ohio. 664 pp., 1194 text figs.

1891. Palaeontology. Preliminary remarks. Advance Sheets, 17th Ann. Rept., Geol. Surv. Indiana, pp. 1-103, 20 pls.

1892a. Palaeontology. Preliminary remarks. 17th Ann. Rept., Indiana Dept. Geol. Nat. Res., pp. 611-705, 20 pls.

1892b. First Appendix, 1892. [North American geology and palaeontology.] Cincinnati, Ohio. Pp. 665-718, text figs. 1195-1265.

1892c. Palaeontology. Advance Sheets, Indiana Geol. Surv., 18th Ann. Rept., 79 pp., 12 pls.

1894. Palaeontology. Indiana Dept. Geol. Nat. Res., 18th Ann. Rept., pp. 257-333, pls. 1-11.

1897. Second appendix to North American geology and palaeontology. Cincinnati, Ohio. Pp. 719-793, text figs. 1266-1458.

Miller, S. A. and Charles Faber

1892. Some new species and new structural parts of fossils. Jour. Cincinnati Soc. Nat. Hist., 15, no. 2, pp. 79-87, pl. 1. 
Mit.lek. S. A. and WM. F. E. Gurl.ey

1894. New genera and species of Echinodermata. Bull. Illinois State Mus. Nat. Hist., 5,53 pp., 5 pls.

1895a. Description of new species of Palaeozoic Echinodermata. Bull. Illinois State Mus. Nat. Hist., 6, 62 pp., 5 pls.

1895b. New and interesting species of Palaeozoic fossils. Bull. Illinois State Mus. Nat. Hist., 7, 89 pp., 5 pls.

MOORE, R. C., ed.

1967. Treatise on Invertebrate Paleontology, Part S, Echinodermata (1), 2 vols., 650 pp., 400 text figs. Geol. Soc. Amer. and Univ. Kansas Press.

Paul, Christopher R. C.

1971. Revision of the Holocystites fauna (Diploporita) of North America. Fieldiana: Geol., 24, 166 pp., 70 text figs.

SCHuchert, Charles

1913. Echinodermata. Cystoidea, pp. 227-248, pls. 31-36, text figs. 6-17. Maryland Geol. Surv. Lower Devonian, text 560 pp., 17 text figs., pls. 1-16. Plates 17-7.3 bound in another volume entitled Devonian Plates.

Shimer, Hervey W. and Robert R. Shrock

1944. Index fossils of North America. New York. 837 pp., 303 pls.

Slocom, Arthur Ware and Aug. F. Foerste

1924. New echinoderms from the Maquoketa Beds of Fayette County, Iowa, lowa Geol. Surv., 29, pp. 315-383, pls. 29-34.

Tillman, C. G.

1967. Triamara cutleri, a new cystoid from the Osgood Formation (Silurian) of Indiana. Jour. Paleontol., 41, no. l, pp. 222-226, l text fig., pl. 25.

WARD, Henry A.

1866. Catalogue of casts of fossils from the principal museums of Europe and America, with short descriptions and illustrations. 228 pp., numerous text figs. 


HAM 
UNIVERSITY OF ILLINOIS-URBANA

$550.5 \mathrm{FI}$

COO1

FIELDIANA, GEOLOGY CHGO

$21-251970 / 72$

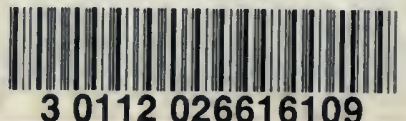

30112026616109 